\title{
Preparation and characterization of a homemade Josephson junction prepared from a thin film sintered in a domestic microwave oven
}

\author{
Gustavo Quereza Freitas ${ }^{a}$, Rafael Zadorosny ${ }^{b}$, Cícero Rafael Cena $^{c}$, João Borsil Silveira ${ }^{b}$, \\ Jéferson Aparecido Moreto ${ }^{a}$, Claudio Luiz Carvalho ${ }^{b}$ \\ ${ }^{a}$ Federal Institute Goiano, Instituto Federal de Educação, Ciência e Tecnologia Goiano, \\ Rio Verde, Goiás, Brazil. \\ ${ }^{b}$ Department of Physics and Chemistry, UNESP - Universidade Estadual Paulista Júlio de Mesquita \\ Filho, Ilha Solteira, São Paulo, Brazil. \\ ${ }^{c}$ Federal Institute of São Paulo, Instituto Federal de Educação, Ciência e Tecnologia de São Paulo, \\ Birigui, São Paulo, Brazil.
}

Received: August 18, 2015; Revised: November 25, 2015; Accepted: December 16, 2015

\begin{abstract}
A homemade Josephson junction was successfully obtained using a superconductor thin film of the BSCCO system. The film was deposited on a lanthanum aluminate, produced from a commercial powder with a nominal composition $\mathrm{Bi}_{1.8} \mathrm{~Pb}_{0.4} \mathrm{Sr}_{2} \mathrm{CaCu}_{2} \mathrm{O}_{\mathrm{x}}$, was thermally treated by a domestic microwave oven. The XRD analysis of the film indicated the coexistence of Bi-2212 and Bi-2223 phases and SEM images revealed that a typical superconductor plate-like morphology was formed. From the electrical characterization, performed using DC four probes technique, it was observed an onset superconducting transition temperature measured around $81 \mathrm{~K}$. At the current-voltage characteristics curve, a step of electric current at zero-voltage could be observed, an indicative that the tunneling Josephson occurred.
\end{abstract}

Keywords: BPSCCO superconductor thin films, Microwave oven, Josephson junction

\section{Introduction}

Since the discovery of the Josephson effect in $1962{ }^{1}$, a high number of studies has been devoted to this effect, after the appearance of High Temperature Superconductors (HTS) ${ }^{2}$, as the $\mathrm{Bi}-\mathrm{Sr}-\mathrm{Ca}-\mathrm{Cu}-\mathrm{O}^{3}$, the research in this area become more attractive due to new phenomenon involved and technological potential applications. The electrical and magnetic behaviours of the HTSs are influenced by the synthesis rote, i.e., dependending on the conection between the grains, some properties like the capacity in transport electrical currents without dissipation is affected. Then, the study of the influence of synthesis routes and heat treatments is of great importance.

Recent studies have been shown a new and alternative method, in terms of thermal treatment of samples, by using a domestic microwave oven as heating source, with the advantage of processing time shorter than conventional methods ${ }^{4}$. Microwave oven has been used as laboratory instrument since 70 's years ${ }^{5}$ and also employed with success in the sintering of metallic oxides and in the crystallization process of thin films $\mathrm{s}^{6,7}$.

The study and understanding of Josephson effect in intergrain junction of BSCCO samples are well established in the literature to samples prepared by conventional methods ${ }^{8}$. Besides the structure and morphological changes observed in materials treated by different routes (varying heating rate, atmosphere, time and temperature) there is no report for samples prepared by domestic microwave oven, in which

*e-mail: gustavoquereza@yahoo.com.br the energy density and heating rate is quite different than conventional methods. The effect and structural differences caused by this new thermal treatment are still been studied in the literature ${ }^{6}$.

The aim of this work was describe the preparation of a bridge-type Josephson junction with length of $200 \mu \mathrm{m}$ and treated thermally in a domestic microwave oven. Then, we used a commercial powder of BSCCO (2212) to produce a superconducting thick film, deposited on a LaAlO3 (100) substrate. The junction properties such as morphology, structure and electrical are extensively studied.

\section{Materials and Methods}

A Josephson junction was obtained from a BPSCCO superconductor film. A commercial powder with nominal composition $\mathrm{Bi}_{1.8} \mathrm{~Pb}_{0.4} \mathrm{Sr}_{2} \mathrm{CaCu}_{2} \mathrm{O}_{\mathrm{x}}$ - $\mathrm{BPSCCO}$ purchase from Superconductive Components Inc. (Lot\#SCI3130SCP3), was employed to produce the film. The powder was spread on a LaAlO3 (100) substrate. The junction was molded by using an adhesive mask, to form a bridge-type junction. The thickness of the film was barelly controled by the mass of the used powder since in this work we focused on the production of a Josepson junction by a microwave heat treatment.

The thermal treatment was carried out using a domestic microwave oven of Panasonic (NN-S 46 BK model, 800W and $2,45 \mathrm{GHz}$ ), by heating the sample at $400{ }^{\circ} \mathrm{C} / 2 \mathrm{~min}$ and then $780{ }^{\circ} \mathrm{C} / 7 \mathrm{~min}$ by using a heating/cooling rate of $20{ }^{\circ} \mathrm{C} / \mathrm{min}$. A small pellet of $\mathrm{SiC}\left(30 \times 25 \times 5 \mathrm{~mm}^{3}\right)$ was used 
as susceptor to improve the microwave energy absorption, and transfer the thermal energy to the film. The temperature control was obtained using a temperature controller with a K-type thermo-couple in contact with the $\mathrm{SiC}$ pellet. Figure 1 shows the experimental scheme for the sample preparation in according to Silveira et al ${ }^{9}$.

The structural characterization of the film was performed by $x$-ray diffraction (XRD), by using a Shimadzu di-ractometer model XRD-6000, in Bragg-Brentano geometry, using a $\mathrm{CuK}_{\alpha 1}$ radiation with wavelength of $1,542 \AA$ and step size of $0.02^{\circ}$. The microstructural and quantitative chemical analysis was carried out using a Zeiss Scanning Electron Microscopy (SEM) model EVO-LS15, with Energy Dispersive of X-ray (EDX) INCA detector.

The resistance versus Temperature (RxT) and Voltage versus Current characteristic curves ( $\mathrm{VxI}$ ) were carried out by using DC four probe method in the Quantum Design PPMS (Physical Properties Magnetic System) equipment.

\section{Results and Discussion}

The commercial powder and the treated film were analyzed by XDR measurements. In Figure 2(a) is shown the XRD patterns for the film which was heat treated in a microwave oven. The down triangles indicate the (2212), the " $\lambda$ ”" symbol indicates the $\mathrm{Ca}_{2} \mathrm{PbO}_{4}$ and the "o" symbol represents the (2223). Then, it can be noted a predominance of the Bi-(2212) phase however, there was also the formation of $\mathrm{Ca}_{2} \mathrm{PbO}_{4}$, a non-superconducting material ${ }^{9,10}$. In Figure 2(b) is shown the XRD analysis of the commercial powder. It can be noted a predominance of $\mathrm{Bi}-(2212)$ phase and the coexistence of two different phases, $\mathrm{Bi}-(2223)$ and $\mathrm{Bi}_{3} \mathrm{~Pb}_{0,4} \mathrm{Sr}_{2} \mathrm{CaCu}_{3} \mathrm{O}_{\mathrm{x}}$ represented by " $\square$ " symbol ${ }^{11-14}$.

Figure 3 shows the microstructure of a bridge-type junction built with $200 \mu \mathrm{m}$ of length and $200 \mu \mathrm{m}$ of width. The microstructure and the morphology of the junction were examined by SEM. The presence of a plate-like structure, typical of the BSCCO system, can be noted. The observed
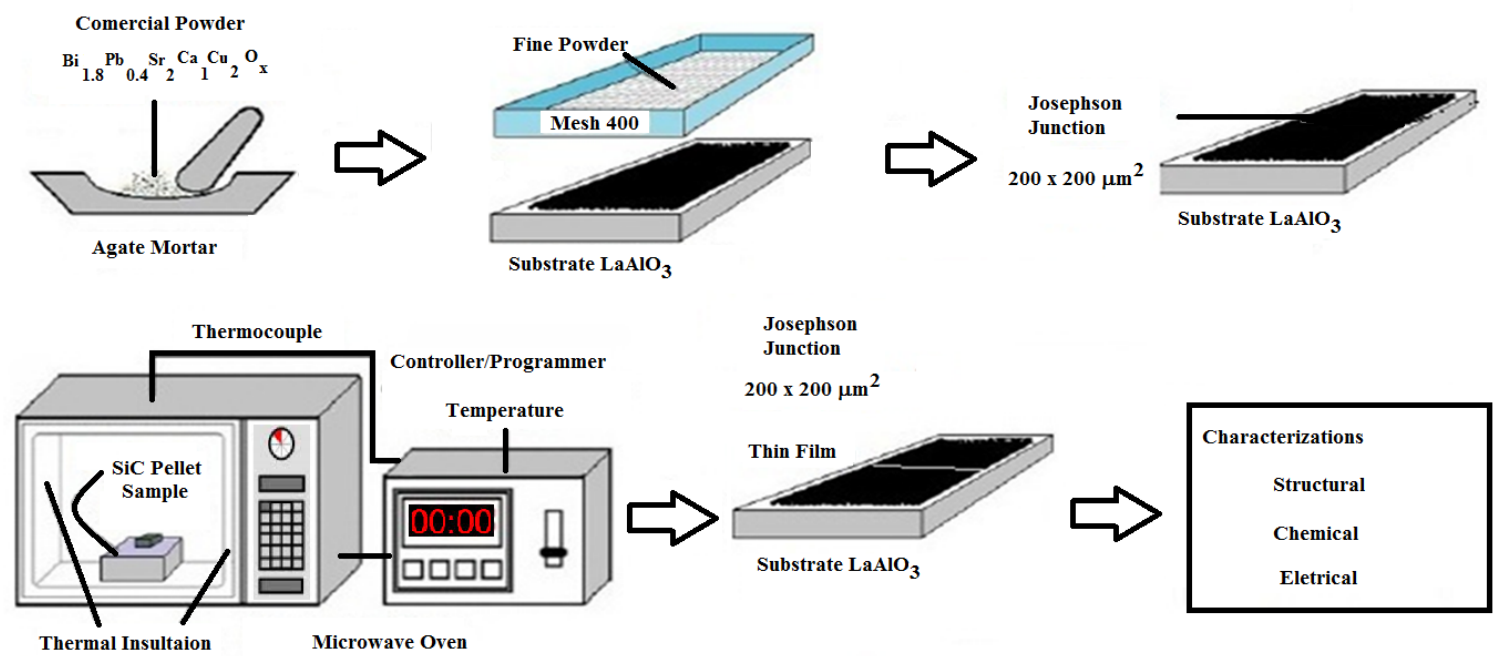

Figure 1: Experimental scheme for the sample preparation [9].
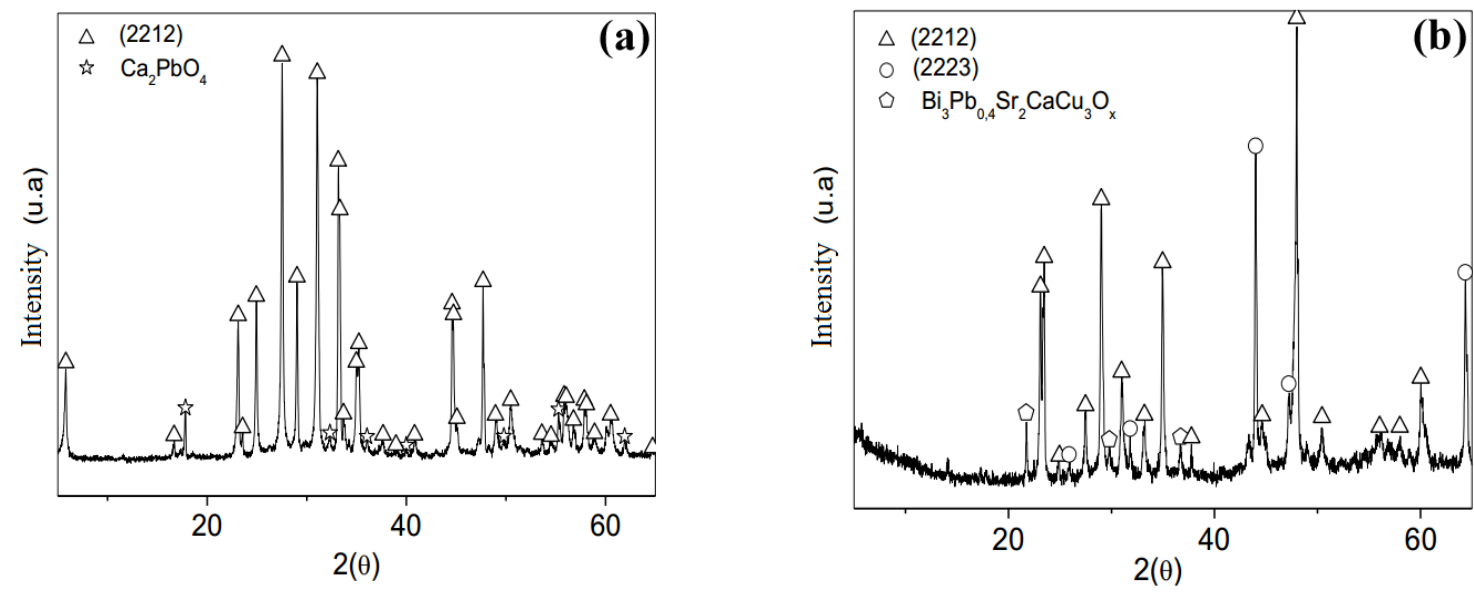

Figure 2: X-ray patterns of BSCCO powder: (a) treated sample (film) by using microwave-oven (b) commercial powder. 
grain coalescence in the microstructure is indicative of the thermal treatment efficacy by using a domestic microwave oven. The chemical composition of the sample was qualitatively analyzed by Energy Dispersive of X-ray (EDX), the results are shown at Table 1. The EDX analysis was performed in two different areas, a smooth area (region 1) and a plate-like area (region 2), as can be seen at Figure 4. The results obtained, point to nominal compositions of $\mathrm{Bi}_{2,18} \mathrm{~Pb}_{0,12} \mathrm{Sr}_{1}$ ${ }_{, 72} \mathrm{Ca}_{0,55} \mathrm{Cu}_{1,53} \mathrm{O}_{9,11}$ to region 1 , and $\mathrm{Bi}_{1,28} \mathrm{~Pb}_{0,9} \mathrm{Sr}_{1,57} \mathrm{Ca}_{0,8} \mathrm{Cu}_{1}$,
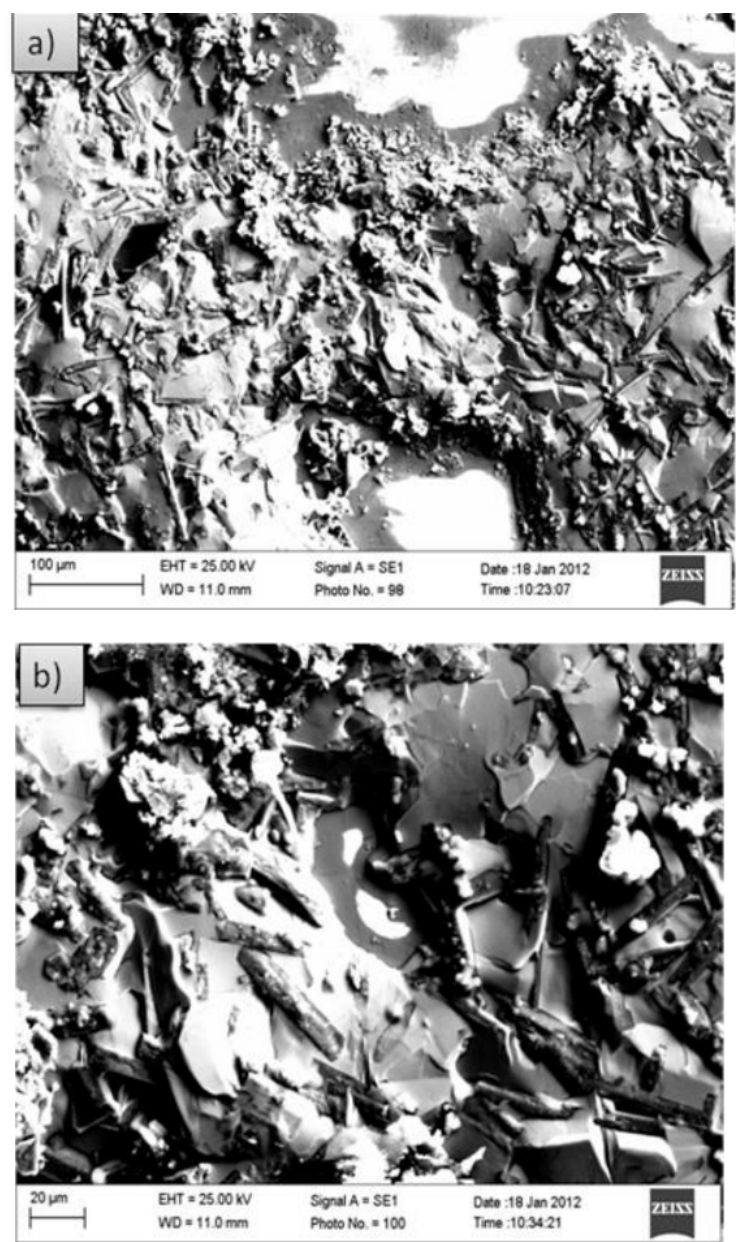

Figure 3: SEM images for the junction builted in the BSCCO film: (a) Junction view, and (b) detailed of the structure observed at the junction.
${ }_{66} \mathrm{O}_{9,8}$ to region 2. Such difference can be attributed to the presence in different contents of $\mathrm{Ca}_{2} \mathrm{PbO}_{4}$ in such regions for example. Basically, a non-superconducting region in the material is desirable since the penetrated magnetic flux (which is originated when an external magnetic field is applied or even due to the self-field produced by the transport current) is trapped in such sites which avoid its dissipative motion.

The electrical resistance as a function of the temperature $(\mathrm{RxT})$ and its respective derivative are show in Figure 5.
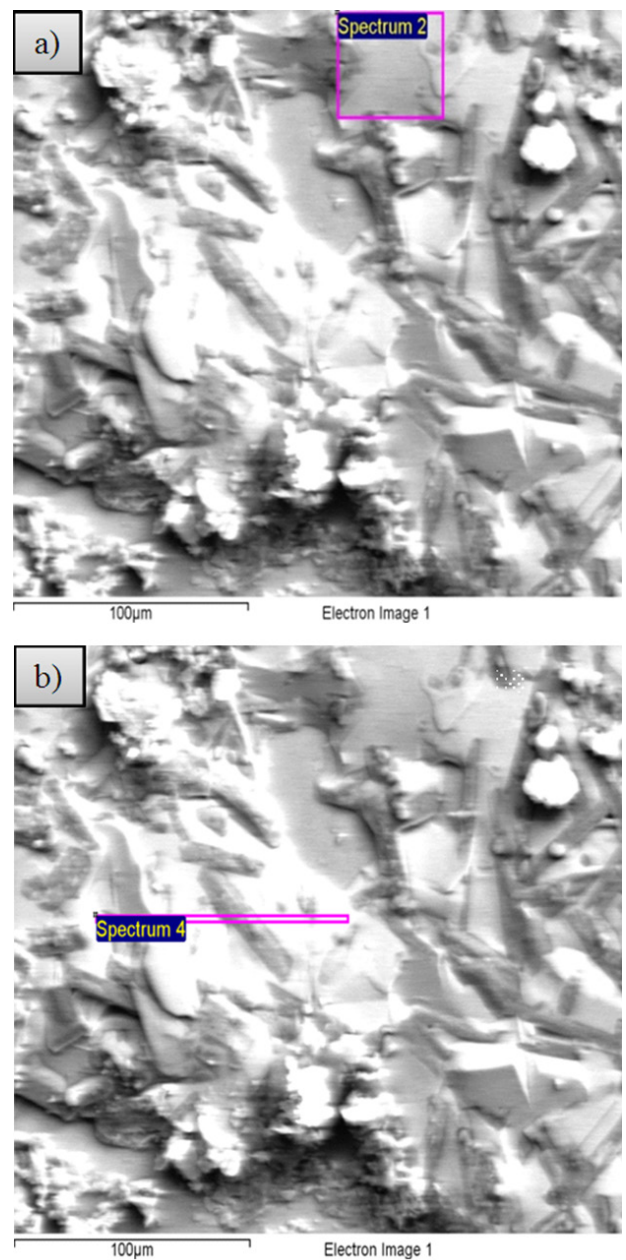

Figure 4: EDS analysis of the different structures presented by the treated sample (a) Smooth and (b) Roughness area.

Table 1: EDS quantitative results of the junction: (a) Smooth and (b) Roughness area.

\begin{tabular}{|c|c|c|c|c|c|c|c|}
\hline Regions & Analysed & \multirow{2}{*}{ Mass \% } & Area (a) & \multirow{2}{*}{ EDS } & \multirow{2}{*}{ Mass \% } & Area (b) & \multirow{2}{*}{ EDS } \\
\hline Element & Nominal & & Atom \% & & & Atom \% & \\
\hline Bi $L$ & 1.8 & 172.92 & 8.43 & 1.28 & 206.82 & 14.36 & 2.18 \\
\hline $\mathrm{PbM}$ & 0.4 & 11.77 & 0.58 & 0.9 & 10.87 & 0.76 & 0.12 \\
\hline $\operatorname{Sr} L$ & 2 & 88.12 & 10.30 & 1.57 & 68.10 & 11.28 & 1.72 \\
\hline $\mathrm{CaK}$ & 1 & 20.59 & 5.96 & 0.80 & 9.97 & 3.61 & 0.55 \\
\hline Си K & 2 & 67.82 & 10.94 & 1.66 & 44.07 & 10.06 & 1.53 \\
\hline$O K$ & 8 & 100.71 & 64.99 & 9.8 & 66.06 & 59.92 & 9.11 \\
\hline Total & 15.2 & 460.93 & 100 & 16.01 & 405.9 & 99.9 & 15.21 \\
\hline
\end{tabular}



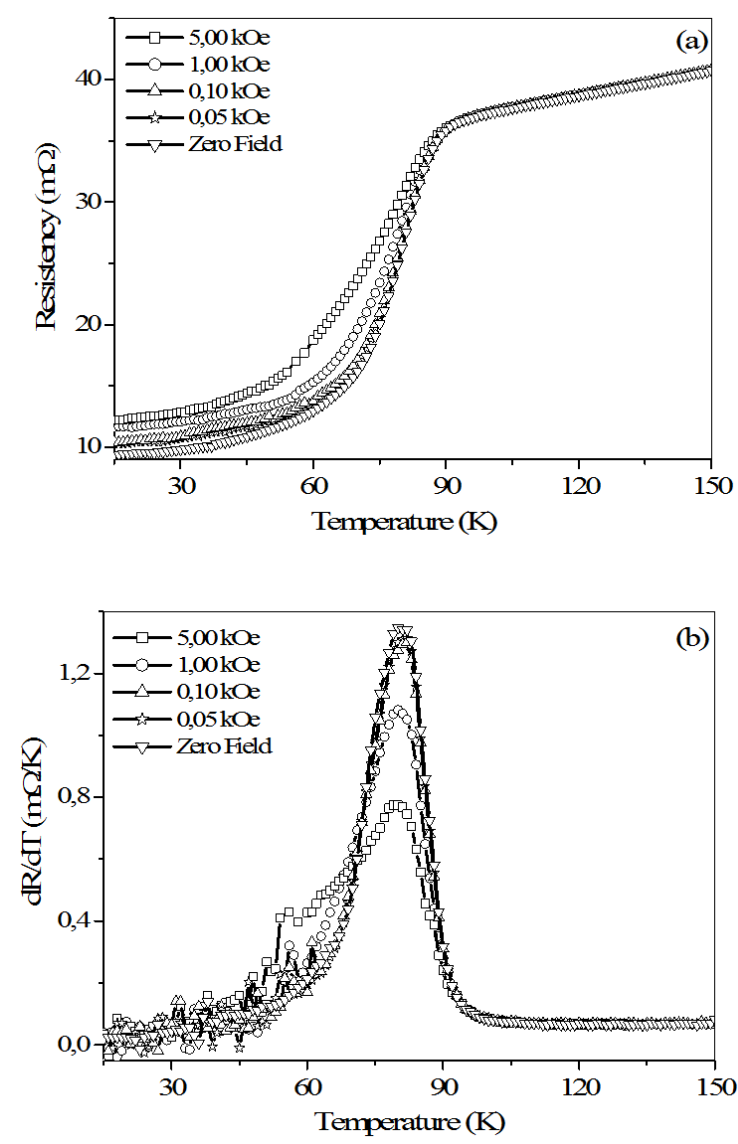

Figure 5: Electrical characterization of the sample to different values of electrical current applied: (a) Electrical resistance versus Temperature, and (b) Derivative curve $\mathrm{dR} / \mathrm{dT}$ versus $\mathrm{T}$.

It can be seen that the sample presents the same critical temperature $\left(\mathrm{T}_{\mathrm{c}}=81 \mathrm{~K}\right)$ for different values of the applied magnetic field, which means that the currents induced by the field are not sufficient to destroy the superconductivity of the junction. It is worth to mention that the $\mathrm{T}_{\mathrm{c}}$ was obtained by the mid-point method, as shown at Figure 5(b). A null resistance was not observed, which can be due to the electrical contacts and also an indication the entire sample is not superconducting yet.

Measurements of voltage as a function of the applied electrical current at different temperatures are shown in Figure 6(a). The change of the slope of the curve at zero voltage confirms the occurrence of the DC Josephson effect even with the sizes of the junction being bigger than coherence length of the material, around $300 \mathrm{~nm}^{15}$. In fact, the Josephson effect observed in this case can be attributed to a sum of such effect which occurs between the grains, i.e., the junction region is small enough to evidence the Josephson effect due to the current that is tunneling between the grains placed there ${ }^{15,16,17}$. Figure 6 (b) shows the derivative curve $(\mathrm{dI} / \mathrm{dV} \times \mathrm{I})$ to demonstrate the electrical current gap. As can be seen in Figure 6 (b) the $\mathrm{V}_{\mathrm{cc}}=0$ Volts indicates the maximum conductance ${ }^{18,19}$. It is very important to note
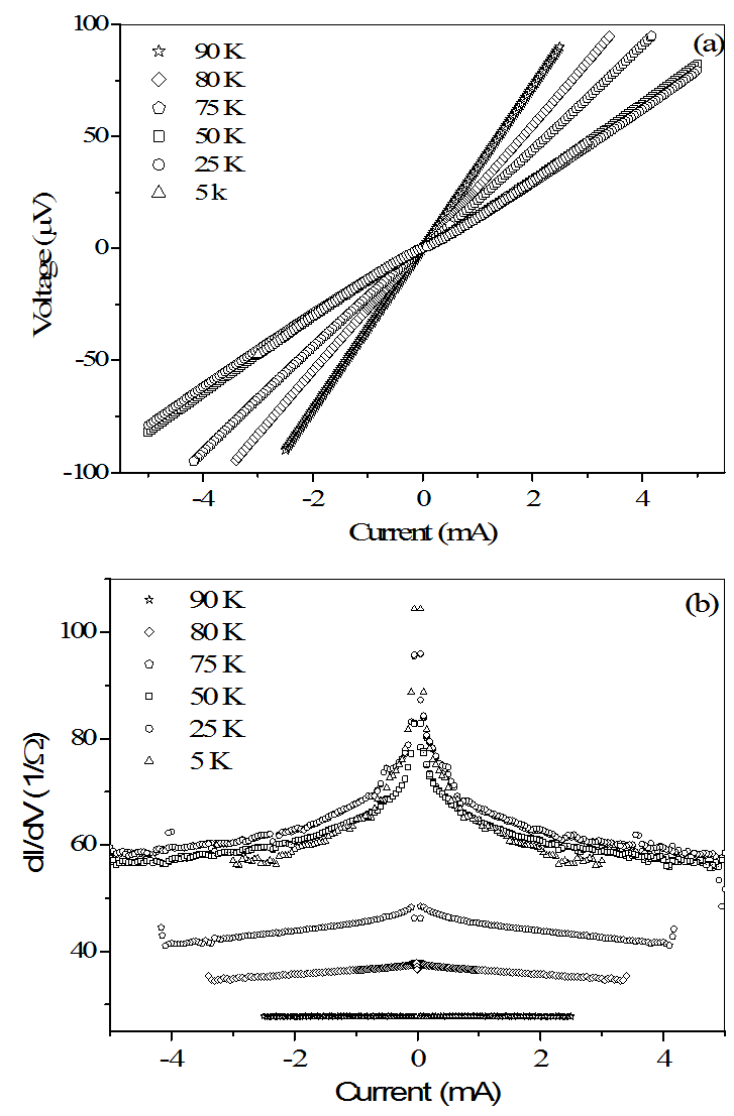

Figure 6: Characteristic curves as function of temperature: (a) Voltage versus electrical current (VxI), and (b) Derivative curve $\mathrm{dI} / \mathrm{dV}$ versus electrical current (I).

that as smaller the temperature higher is the current gap (dI/ dV) according to Argaman ${ }^{20}$.

\section{Conclusions}

A Josephson junction was successfully obtained in a superconducting film, which was heat treated by using a domestic microwave oven. The electrical characterization of the sample showed a superconductor transition temperature around $\mathrm{T}_{\mathrm{c}}=81 \mathrm{~K}$. The IxV curves show a change of slope at zero-voltage, which is characteristic of the Josephson effect. Finally, a residual resistance showed to increases with the magnetic field.

\section{Acknowledgments}

This work was supported by FUNDUNESP, PROPe, $\mathrm{CNPq}$ and Capes. The authors would like to thank the Polymer Group by the XRD measurements and Professor Dr. João Carlos S. de Moraes by the SEM/EDX measurements both located in the Physics and Chemistry Department; Professor Dr. Sebastião Ribeiro from EEL-USP by the microwave susceptors and Professor Dr. Wilson Aires Ortiz, leader of the "Superconductivity and Magnetism Group" of UFSCar by discussions. 


\section{References}

1. Josephson BD. Possible new effects in superconductive tunnelling. Physics Letters. 1962. 1(7): 251-253. doi:10.1016/00319163(62)91369-0

2. Bednorz JG, Muller KA. Possible high- $T_{c}$ superconductivity in the Ba-La-Cu-O system. Zeitschrift Fur Physik B-Condensed Matter: 1986;64(2):189-193.

3. Maeda H, Tanaka Y, Fukutom M, Asano T. A new high-Tc oxide superconductor without a rare-earth element. Japanese Journal of Applied Physics Part 2-Letters. 1988;27(2):L209-L210.

4. Rao KJ, Vaidhyanathan M, Ramakrishnan PA. Synthesis of inorganic solids using microwaves. Chemistry of Materials. 1999;11(4):882-895.

5. Keyson D, Longo E, Vasconcelos JS, Varela JA, Éber S, DerMaderosian A. Synthesis and ceramics processing by domestic microwave oven. Cerâmica. 2006;52(321):50-56. http://dx.doi.org/10.1590/S0366-69132006000100007

6. Menezes RR. Microwave sintering of ceramics. Part II: Sintering of $\mathrm{ZnO}-\mathrm{CuO}$ varistors, ferrite and porcelain bodies. Cerâmica. 2007;53:108-115. http://dx.doi.org/10.1590/S036669132007000200002

7. Kittel C. Introduction to solid state physics. 8th. Hoboken, NJ: Wiley; 2005.

8. Kleiner R, Steinmeyer F, Kunkel G, Müller P. Intrinsic Josephson effects in $\mathrm{Bi}_{2} \mathrm{Sr}_{2} \mathrm{CaCu}_{2} \mathrm{O}_{8}$ single-crystals. Physical Review Letters. 1992;68(15):2394-2397.

9. Silveira JB, Carvalho CL, Torsoni GB, Aquino HA, Zadorosny R. Thermal treatment of superconductor thin film of the BSCCO system using domestic microwave oven. Physica $C$. 2012;478:56-59. doi:10.1016/j.physc.2012.03.021

10. Abdolhosseini I, Kameli P, Salamati H. The effect of precursor powder size on the microstructure and intergranular properties of $(\mathrm{Bi}, \mathrm{Pb})(2) \mathrm{Sr}(2) \mathrm{Ca}(2) \mathrm{Cu}(3) \mathrm{O}(\mathrm{y})$ superconductors. Japanese Journal of Applied Physics. 2008;47(6):4505-4510.

11. Wong-Ng W, Chiang CK, Freiman SW, Cook LP. Phase formation of high- $\mathrm{T}_{\mathrm{c}}$ superconducting oxides in the $\mathrm{Bi}-\mathrm{Pb}-\mathrm{Sr}-\mathrm{Ca}-\mathrm{Cu}-\mathrm{O}$ glass. American Ceramic Society Bulletin. 1992;71(8):12611266.

12. Pandey D, Mahesh R, Singh AK, Tiwari VS, Kak S K. On the formation of the 2223-phase in the $\mathrm{Bi}-\mathrm{Pb}-\mathrm{Sr}-\mathrm{Ca}-\mathrm{Cu}-\mathrm{O}$ system using $\mathrm{Pb}_{0.2} \mathrm{SrCa}\left(\mathrm{Co}_{3}\right)_{2.2}$ precursors. Solid State Communications. 1990;76(5):655-658.

13. Pandey D, Mahesh R, Singh AK, Tiwari VS, Kak SK. Zero resistivity at $122-\mathrm{K}$ in $\mathrm{Bi}-\mathrm{Pb}-\mathrm{Sr}-\mathrm{Ca}-\mathrm{Cu}-\mathrm{O}$ system. Solid State Communications. 1990;75(6):499-502. doi:10.1016/00381098(90)90485-T

14. Sharma SV, Sinha G, Nath TK, Chakroborty S, Majumdar AK. Superconducting fluctuation study of the $110-\mathrm{K}$ phase in polycrystalline $\mathrm{Bi}_{1.6} \mathrm{~Pb}_{0.4} \mathrm{Sr}_{2} \mathrm{Ca}_{2} \mathrm{Cu}_{3} \mathrm{O}_{\mathrm{y}}$ high- $\mathrm{T}_{\mathrm{c}}$ superconductor. Physica C. 1995;242(3-4):351-359. doi:10.1016/09214534(94)02405-7

15. Kataria ND, Tomar VS, Gupta AK, Kumar M. Josephson effects in a constricted Bi-Sr-Ca-Cu-O bulk superconductor. Journal of Physics C-Solid State Physics. 1988;21(15):L523-L527.

16. Hamanaka K, Oho K, Tachiki T, Uchida, T. Intrinsic Josephson Junctions in Bi-2212 thin films fabricated by metal-organic decomposition. Ieee Transactions on Applied Superconductivity. 2011;21(3):168-171.

17. Chu CW, Bechtold J, Gao L, Hor PH, Huang ZJ, Meng $\mathrm{RL}$, et al. Superconductivity up to $114-\mathrm{K}$ in the Bi-Al-Ca-Sr$\mathrm{Cu}-\mathrm{O}$ compound system without rare-earth elements. Physical Review Letters. 1988;60(10):941-943.

18. Tinkham M. Introduction to superconductivity. 2nd. New York: McGraw Hill; 1996.

19. Lehnert KW, Argaman N, Blank HR, Wong KC, Allen SJ, Hu $\mathrm{EL}$, et al. Nonequilibrium supercurrents in mesoscopic NbInAs-Nb junctions. Microelectronic Engineering. 1999;47(14):377-379. doi:10.1016/S0167-9317(99)00238-5

20. Argaman N. Nonequilibrium Josephson-like effects in wide mesoscopic SNS junctions. Superlattices and Microstructures. 1999;25(5-6):861-875. doi:10.1006/spmi.1999.0724 